\title{
Apelin plays a catabolic role on articular cartilage: In vivo and in vitro studies
}

\author{
PENG-FEI HU, WEI-PING CHEN, JING-LI TANG，JIA-PENG BAO and LI-DONG WU \\ Department of Orthopedic Surgery, The Second Hospital of Medical College, \\ Zhejiang University College of Medicine, Hangzhou, P.R. China
}

Received March 31, 2010; Accepted May 17, 2010

DOI: 10.3892/ijmm_00000473

\begin{abstract}
Adipokines play key roles in the regulation of bone growth, obesity, diabetes mellitus type 2, and HIV infection. As a newly discovered hormone in the adipokine family, the precise role of apelin on articular cartilage metabolism is not yet clear. The aim of this study was to evaluate the role of apelin on articular cartilage. In vitro, we examined the effects of apelin on normal chondrocyte proliferation and gene expression of metalloproteinases (MMPs) and interleukin-1ß (IL-1ß). In vivo, by intra-articular injection with apelin, we examined MMP-3, -9, collagen II and IL-1ß at both gene and protein levels. Furthermore, we measured the messenger RNA (mRNA) expression of ADAMTS-4 and -5 (a disintegrin and metalloproteinase with thrombospondin motifs 4 and 5) and the proteoglycan content in articular cartilage. Apelin stimulated the proliferation of chondrocytes and significantly increased mRNA levels of MMP-1, $-3,-9$ and IL-1ß in vitro. Intra-articular injection with apelin in vivo up-regulated the expression of MMP-3, -9 , and IL-1ß as well as decreased the level of collagen II. Additionally, after treatment with apelin, mRNA levels of ADAMTS-4 and -5 markedly increased and depletion of proteoglycan in articular cartilage was found by histological assessment. These findings suggest that apelin plays a catabolic role in cartilage metabolism and is a risk factor in the pathophysiology of osteoarthritis.
\end{abstract}

\section{Introduction}

Osteoarthritis (OA), a worldwide degenerative joint disease in elderly individuals, frequently affects the hands, knees, hips, and spine. It can lead to severe symptoms such as, pain, malformation of joints and disabilities (1). To date, articular cartilage breakdown, osteophyte formation, subchondral sclerosis, and alterations of the synovium were considered as the major pathological changes in the progression of OA (2).

Correspondence to: Professor Li-dong Wu, Department of Orthopedic Surgery, The Second Hospital of Medical College, Zhejiang University, JieFang Road 88\#, 310009 Hangzhou, P.R. China

E-mail: 1dwu@yahoo.com

Key words: apelin, osteoarthritis, adipokines, chondrocytes
Although the exact mechanism of OA is still unclear, the main risk factors known to affect the progression of OA have been extensively investigated, such as age, gender, injury, and obesity. Previous studies have demonstrated that $\mathrm{OA}$ is strongly correlated with a high body mass index (BMI) and weight loss is an effective way to decrease progression of OA (3). Regarding weight-affording joints, such as the knee and hip, high mechanical stress is a sufficient explanation for obesity-induced OA (4). However, a prospective study presented that obesity is also a risk factor for the incidence of OA of the hand (5). As a non-weight bearing joint, excess physical stress cannot explain this phenomenon. A novel family of cytokines named adipokine, secreted by white adipose tissue (WAT), was viewed as a potential link between obesity and OA. Increasing evidence suggests that adipokines, including leptin, adiponectin, resistin, and visfatin, exert proinflammatory and catabolic/anabolic roles during the pathophysiology of OA (6-10).

Apelin was identified as a new member in the adipokine family from bovine stomach extracts in 1998 (11). It was synthesized as a prepropeptide consisting of 77 amino acids and can be cleaved into multiple active molecular forms including apelin-36 and -13 (12). The biological activity of apelin is mediated by its specific receptors named APJ, a G-protein-coupled receptor with seven transmembrane domains (13). The physiological and pathophysiological roles of the apelin system are observed in a variety of aspects. Firstly, in the cardiovascular system, apelin can protect the heart against ischemia reperfusion injury and down-regulate blood pressure through nitric oxide-dependent arterial vasodilatation (14). Secondly, apelin can stimulate proliferation of vascular smooth muscle cells and human osteoblasts via a certain signal transduction pathway $(15,16)$. Furthermore, apelin induces fluid homeostasis by decreasing the circulating plasma antidiuretic hormone (ADH) levels after intra-cerebroventricular administration of apelin-13 (17). The apelin system has been observed in the pathophysiology of many diseases, such as obesity, diabetes mellitus type 2, HIV infections, and osteoporosis (18-20). In addition, Han et al demonstrated that LPS, IL-6, or interferon- $\gamma$ (IFN- $\gamma$ ) treatment can induce the increasing expression of enteric apelin in rodents, suggesting a potential link between apelin and inflammation (21). These data indicate that the apelinAPJ system is important in the regulation of various physiological and pathological procedures. 
Recently, Liao and colleagues demonstrated that apelin and its receptor were expressed in human osteoblasts. They demonstrated that apelin can induce osteoblast proliferation through the APJ/PI3k/Akt pathways and protect osteoblasts against apoptosis $(15,22,23)$. These findings suggest that apelin plays a key role in bone metabolism. To date, nothing is known about the role of apelin in cartilage metabolism. The aim of the present study was to disclose the role of apelin on articular cartilage. First, we evaluated the effect of apelin on normal chondrocyte proliferation and mRNA levels of MMP-1, -3, -9 and IL-1ß in vitro. Secondly, we assessed the effect of apelin by intra-articular injection with apelin and examined MMP-3, -9, collagen II and IL-1ß at both gene and protein levels in vivo. Also, we measured mRNA expression of ADAMTS-4 and -5 and the proteoglycan content in articular cartilage after apelin treatment.

In the present study, we found that apelin stimulated the proliferation of chondrocytes and significantly increased mRNA levels of MMP-1, -3, -9 and IL-1ß in vitro. Intraarticular injection with apelin in vivo up-regulated the expression of MMP-3, -9 , and IL-1ß as well as decreased the level of collagen II at both gene and protein levels. Additionally, after the apelin treatment, the mRNA levels of ADAMTS-4 and -5 showed a marked increase, and depletion of proteoglycan in articular cartilage as exhibited by histological assessment.

\section{Materials and methods}

Primary cultures of normal rat articular chondrocytes. Rat articular chondrocytes for primary culture were isolated from knee joints of 4-week-old Sprague-Dawley rats (The Animal Center of Zhejiang University, Hangzhou, China). Cartilages were washed in phosphate buffered saline (PBS) and finely minced into $\sim 1-2 \mathrm{~mm}^{3}$ pieces. Chondrocytes were released from articular cartilage after being digested for $0.5 \mathrm{~h}$ with $0.2 \%$ pronase (Sigma, St. Louis, MO, USA), followed by digestion for $4 \mathrm{~h}$ with $0.1 \%$ collagenase (Sigma) at $37^{\circ} \mathrm{C}$. After collection of individual cells by centrifugation, cells were cultured in Dulbecco's modified Eagle medium (DMEM) supplemented with antibiotic-antimycotic solution $(100 \mathrm{U} / \mathrm{ml}$ penicillin, $100 \mu \mathrm{g} / \mathrm{ml}$ streptomycin, and $0.25 \mu \mathrm{g} / \mathrm{ml}$ amphotericin B; Life Technologies). Medium was replaced every 2 days, and cells were incubated at $37^{\circ} \mathrm{C}$ under a humidified $5 \% \mathrm{CO}_{2}$ atmosphere. The use of rats for this study was approved by the University of Zhejiang Institutional Animal Care and Use Committee, Hangzhou, China.

Assessment of cell proliferation. Chondrocytes were plated on a 96-well plate at a density of $2 \times 10^{4}$ cells $/ \mathrm{cm}^{3}$ and chondrocyte proliferation was assessed in the presence of increasing concentrations of apelin $(0,0.5,1,10,100 \mathrm{nM})$ (Phoenix Peptides, Burlingame, CA, USA), by using a 3-[4, 5dimethylthiazol-2-yl]-2, 5-diphenyltetrazolium bromide (MTT) assay (Sigma, St. Louis, MO, USA). Cell proliferation was examined at 24 and $48 \mathrm{~h}$ after addition of apelin.

mRNA expression of IL-1 $\beta, M M P-1,-3$ and -9 by real-time quantitative PCR. Rat articular chondrocytes were plated on a 6-well plate at a density of $5 \times 10^{4}$ cells $/ \mathrm{cm}^{3}$ and stimulated with apelin $(0,0.5,1,10,100 \mathrm{nM})$ for $48 \mathrm{~h}$. Then we separated the supernatant, centrifuged and stored cells and supernatants at $-80^{\circ} \mathrm{C}$ until use. Samples were pulverized in liquid nitrogen, and total RNA was isolated using Tri reagent (Sigma) according to the manufacturer's instructions. After treatment for $20 \mathrm{~min}$ at $37^{\circ} \mathrm{C}$ with 1 unit of DNase I (Sigma) to prevent genomic DNA contamination, $1 \mu \mathrm{g}$ of total RNA was reverse transcribed using $10 \mathrm{pmol}$ of random hexanucleotidic primers (Promega, USA), $0.5 \mathrm{mM}$ dNTPs, and 200 units of Moloney murine leukemia virus reverse transcriptase (Promega) at $37^{\circ} \mathrm{C}$ for $1 \mathrm{~h}$. The reaction was stopped by incubation at $70^{\circ} \mathrm{C}$ for $10 \mathrm{~min}$. Then, quantification of gene expression levels for targeted genes were carried out by real-time quantitative PCR with the iCycler apparatus system (Bio-Rad, USA). $\mathrm{iQ}^{\mathrm{TM}}$ SYBR Green supermix PCR kit (Bio-Rad) was used for real-time monitoring of amplification ( $5 \mathrm{ng}$ of template cDNA, 45 cycles, $95^{\circ} \mathrm{C} / 15 \mathrm{sec}, 60^{\circ} \mathrm{C} / 15 \mathrm{sec}$ ) with primers in Table I. A parallel amplification of RatGAPDH (NM_017008), 5' GGT GGACCTCATGGCCTACAT 3' and 5' GCCTCTCTCTTGC TCTCAGTATCCT 3' primers, was carried out to normalize expression data of the targeted gene transcript. The relative level of targeted gene expression is calculated for 100 copies of the $18 \mathrm{~s}$ housekeeping gene following the formula,

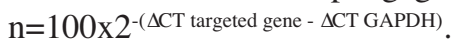

Intra-articular injection of rat with r-apelin. In order to investigate the effect of apelin on articular cartilage in vivo, 36 male Sprague-Dawley rats (250 g) (The Animal Center of Zhejiang University, Hangzhou, China) were used. Before the experiment, animals were acclimatized to the laboratory environment for 7 days under controlled temperature and lighting conditions, and received food and water ad libitum. Then rats were evenly divided into two groups at random, control and apelin groups. After they were anesthetized with $10 \%$ chloral hydrate (Sigma-Aldrich), rats from apelin group were injected in the right knee joint with $50 \mu 1$ of $1 \mathrm{nM}$ recombinant rat apelin (Phoenix Peptides). The control group was injected with $50 \mu 1$ sterile saline. This study was approved by the University of Zhejiang Institutional Animal Care and Use Committee, Hangzhou, China.

Evaluation of proteoglycan content in articular cartilage by Safranin $O$ staining. Twelve rats (6, apelin group; 6, control group) were sacrificed $48 \mathrm{~h}$ after intra-articular injection. Specimens of tibial plateaus were fixed in $10 \%$ neutral buffered formalin for $48 \mathrm{~h}$, placed in 5\% hydrochloric acid for $24 \mathrm{~h}$, dehydrated in alcohol sequentially, embedded into paraffin blocks and cut into sections of $5 \mu \mathrm{m}$. Sections were stained with Safranin O for cartilage proteoglycan content evaluation. Three sagittal sections of each tibial plateau were used for evaluation.

Real-time PCR analysis of MMP-1, -3, -9, IL-1 $\beta$, collagen II, ADAMTS-4 and -5. Twelve rats (6, apelin group; 6, control group) were sacrificed $48 \mathrm{~h}$ after intra-articular administration of apelin, and full-thickness pieces of the articular cartilage of the tibial plateau and femoral condyle were collected aseptically. mRNA expression of MMP-1, -3, -9, IL-1ß, collagen II, ADAMTS-4 and -5 were analyzed by real-time quantitative PCR as stated above (2.3). The primers are listed in Table I. 
Table I. Primers of targeted genes.

\begin{tabular}{|c|c|c|c|c|}
\hline Gene & GenBank & Primer sequences & Size (bp) & Annealing $\left({ }^{\circ} \mathrm{C}\right)$ \\
\hline MMP-1 & NM_001134530 & $\begin{array}{l}\text { F: 5-GACGTGGACCGACAACAGTGA-3 } \\
\text { R: 5-GGGGAACATTAGTGCTCCTACATC-3 }\end{array}$ & 112 & 60 \\
\hline MMP-3 & NM_133523 & $\begin{array}{l}\text { F: 5-CTGGGCTATCCGAGGTCATG-3 } \\
\text { R: 5-TGGACGGTTTCAGGGAGGC-3 }\end{array}$ & 77 & 60 \\
\hline MMP-9 & NM_031055 & $\begin{array}{l}\text { F: 5-ACCCCATGTATCACTACCACGAG-3 } \\
\text { R: 5-TCAGGTTTAGAGCCACGACCAT-3 }\end{array}$ & 91 & 60 \\
\hline IL-1ß & NM_031512 & $\begin{array}{l}\text { F: 5-CCTAGGAAACAGCAATGGTCGGGAC-3 } \\
\text { R: 5-GTCAGAGGCAGGGAGGGAAACAC-3 }\end{array}$ & 239 & 62 \\
\hline ADAMTS-4 & NM_023959 & $\begin{array}{l}\text { F: 5-GTGGCAGATGACAAGATGGCAGCATT-3 } \\
\text { R: 5-GTTGACAGGGTTTCGGATGCTTGGAT-3 }\end{array}$ & 117 & 60 \\
\hline ADAMTS-5 & AY382879 & $\begin{array}{l}\text { F: 5-GTGATGACCATGAGGAGCACTACGAT-3 } \\
\text { R: 5-GAACATATGGTCCCAACGTCTGCCATT-3 }\end{array}$ & 112 & 60 \\
\hline COL II & L48440 & $\begin{array}{l}\text { F: 5-CTGGTGGAGCAGCAAGAGC-3 } \\
\text { R: 5-GTGGACAGTAGACGGAGGAAAG-3 }\end{array}$ & 144 & 60 \\
\hline Rat GAPDH & NM_017008 & $\begin{array}{l}\text { F: 5-GGTGGACCTCATGGCCTACAT-3 } \\
\text { R: 5-GCCTCTCTCTTGCTCTCAGTATCCT-3 }\end{array}$ & 88 & 60 \\
\hline
\end{tabular}
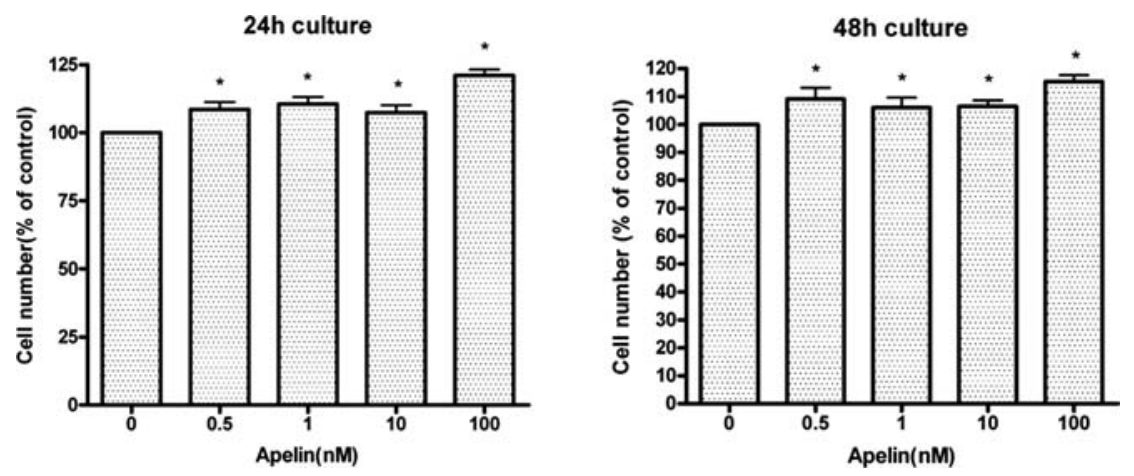

Figure 1. The effects of apelin on chondrocyte proliferation as determined by the MTT assay. Chondrocytes $(5,000)$ were initially plated in each well of a 96-well plate and treated with apelin at $0,0.5,1,10,100 \mathrm{nM}$. At 24 and $48 \mathrm{~h}$, the number of viable cells was measured by MTT assay. Values are mean \pm SD of three independent experiments. "Statistical significance in viable cell numbers $(\mathrm{P}<0.05)$.

Western blot analysis of MMP-3, -9, IL-1 $\beta$ and collagen II. The tibial plateaus and femoral condyle were collected from 12 rats (6, apelin group; 6, control group) and washed with iced PBS. Protein levels of MMP-3, -9, IL-1ß and collagen II were measured as previously described (24). Antibodies to MMP-3, -9, IL-1ß, collagen II and B-actin were obtained from Santa Cruz Biotechnology. Immunoblotting was performed using appropriate antibodies and then with horseradish peroxidase (HRP)-conjugated secondary antibody diluted at 1:2,000 in TBS, 0.1\% Tween-20 and 5\% BSA. Protein expression levels were detected using ECL (GE Healthcare) and BioMaxfilm (Kodak). The relative variations between the bands of the apelin treatment samples and control group were calculated using the same image.

Statistical analysis. All data are expressed as mean \pm standard deviation (SD). Statistical analyses were performed with software, SPSS 12.0 for Windows. Statistical significance was determined using Student's t-test. P-values $<0.05$ were considered significant.

\section{Results}

Effects of apelin on chondrocyte proliferation. Effects of apelin on normal chondrocyte proliferation were examined at apelin concentrations of $0.5,1,10,100 \mathrm{nM}$ after 24 and $48 \mathrm{~h}$ of culture (Fig. 1). Chondrocytes at every concentration proliferated actively. Significant differences were observed between cells treated with different apelin concentrations at different times $(\mathrm{P}<0.05)$. These results demonstrate that apelin stimulates proliferation of chondrocytes at any of these concentrations.

Effects of apelin on gene expression of IL-1 $\beta, M M P-1,-3$ and -9 in vitro. After treated with apelin for $48 \mathrm{~h}$, we detected the mRNA levels of IL-1ß, MMP-1, -3 and -9 using real-time 

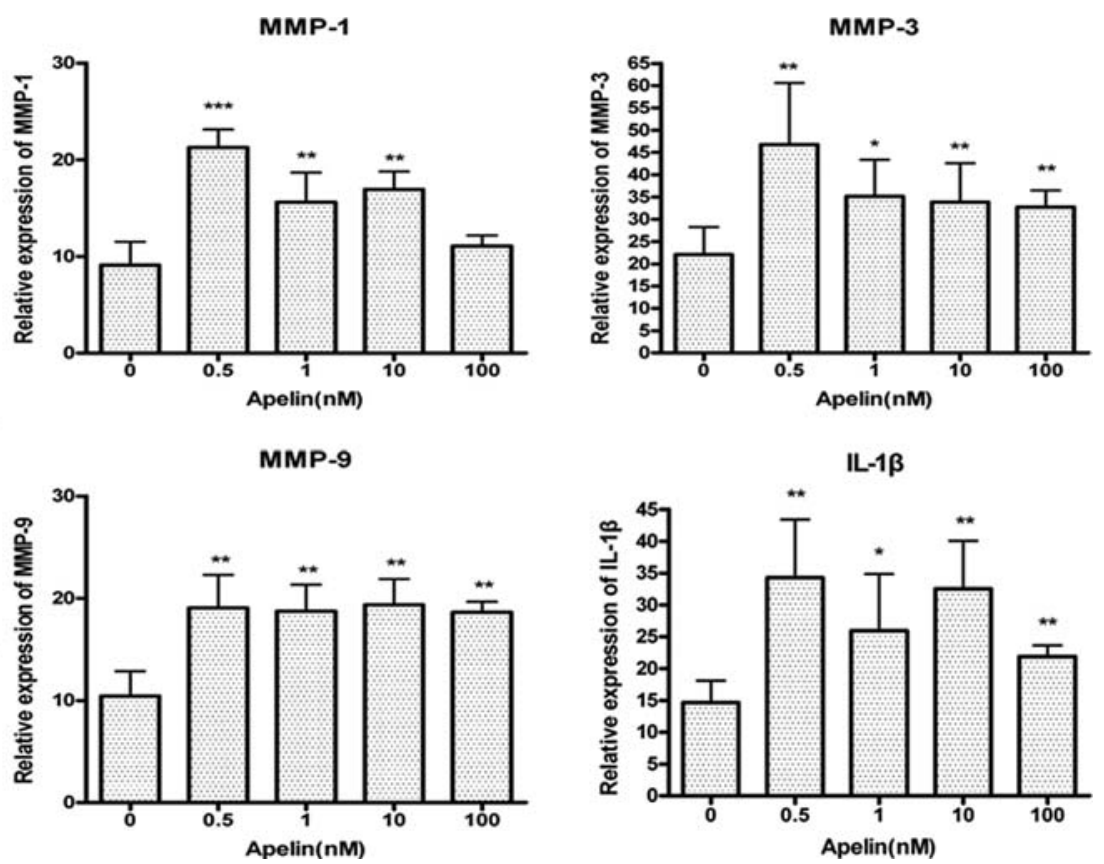

Figure 2. Effects of apelin on gene expression of MMP-1, -3, -9, and IL-1ß. mRNA expression levels of MMP-1, $-3,-9$ and IL-1ß were up-regulated significantly after apelin $(0.5,1,10,100 \mathrm{nM})$ treatment. ${ }^{*} \mathrm{P}<0.05,{ }^{* *} \mathrm{P}<0.01,{ }^{* * *} \mathrm{P}<0.001$ compared with apelin $0 \mathrm{nM}$.
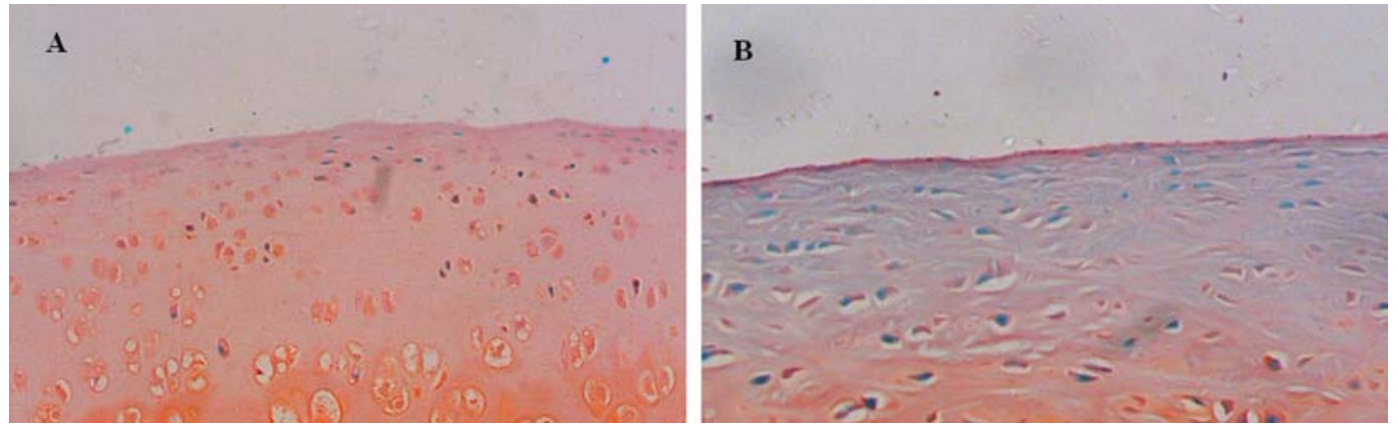

Figure 3. Safranin O and fast green stained sections of tibial plateaus of control group (A) and apelin group (B). According to some typical sections, depletion of aggreacan was apparent from the superficial zone to the middle zone after apelin treatment. Original magnification x 100 .

quantitative PCR. As noted in Fig. 2, apelin $(0.5,1,10$, $100 \mathrm{nM})$ significantly increased the transcript levels of the catabolic factors MMP-1, $-3,-9$ and also the proinflammatory cytokine, IL-1ß.

Gross histological evaluation. The tibial plateaus after intraarticular injection of apelin $48 \mathrm{~h}$ later exhibited a decrease in Safranin O staining compared to control group. These results indicate the content of cartilage proteoglycan reduced after apelin administration. According to some typical sections of tibial plateaus (Fig. 3), depletion of aggreacan was observed from the superficial to the middle zone after apelin treatment.

Effects of apelin on gene expression of IL-1 $\beta, M M P-1,-3,-9$, ADAMTS-4, -5, and collagen II in vivo. Using real-time quantitative PCR we observed that gene levels of the catabolic factors, such as MMP-1, -3, -9, ADAMTS-4 and -5 were significantly increased after apelin treatment (Fig. 4). Proinflammatory cytokine, IL-1ß was also remarkably up- regulated. Meanwhile, the mRNA abundance of collagen II was at a low level in the apelin group.

Effects of apelin on protein syntheses of MMP-3, -9, collagen II and $I L-1 \beta$. Protein syntheses of MMP-3, -9, collagen II and IL- $1 \beta$ in tibial plateaus and femoral condyle from normal controls and intra-articular apelin treatment groups were analyzed by Western blotting (Fig. 5). All target proteins exhibited bands at their corresponding position in the study. Protein syntheses of MMP-3, -9, and IL- 13 increased in the tissues after apelin treatment. Collagen II decreased significantly in the cartilage by apelin treatment compared with the normal samples. These results are consistent with the findings to date of real-time quantitative PCR.

\section{Discussion}

To date, little is known about apelin in the regulation of chondrocytes. As a newly discovered adipokine, we aimed to 


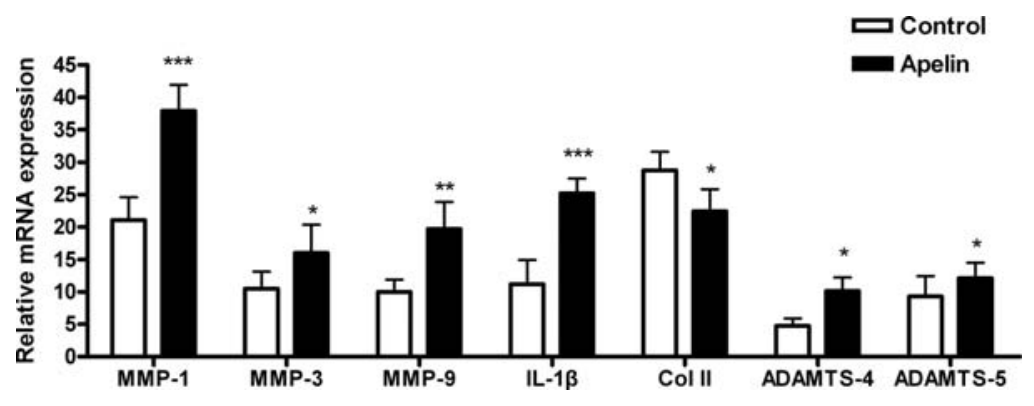

Figure 4. Effects of apelin on the gene expressions of the IL-1ß, MMP-1, -3, -9, collagen II, ADAMTS-4 and -5 in vivo. After intra-articular injection of rats with apelin $(1 \mathrm{nM})$, catabolic factors, including MMP-1, -3, -9, ADAMTS-4 and -5 , and proinflammatory cytokine likes IL-1ß were significantly increased. The gene expression of collagen II was reduced in the apelin group. ${ }^{*} \mathrm{P}<0.05,{ }^{*} \mathrm{P}<0.01,{ }^{* * *} \mathrm{P}<0.001$ compared with control group.

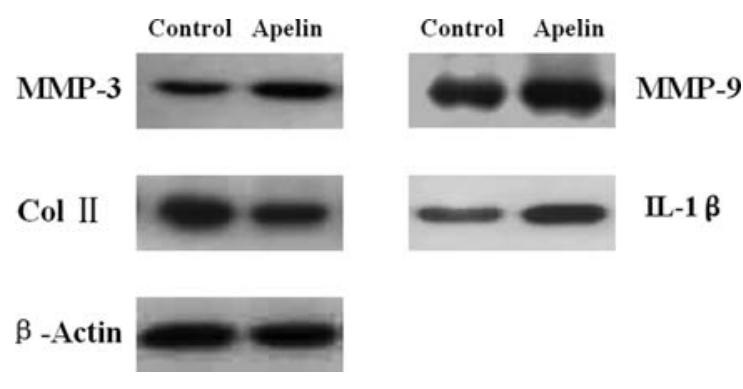

Figure 5. Western blot of the protein syntheses of MMP-3, -9, collagen II and IL-1ß in articular cartilage from control and apelin treatment samples. Experiments were performed in triplicate and the bands shown represent typical results. All these target proteins exhibited bands at their corresponding position. After treated with apelin $(1 \mathrm{nM})$ for $48 \mathrm{~h}$, the protein levels of MMP-3, -9 , and IL-1ß significantly increased, and synthesis of collagen II decreased.

reveal whether apelin has a key role in the development of OA. To address this problem, we designed this experiment into two parts: i) evaluation of the effects of apelin on normal chondrocyte proliferation and expression of MMP-1, -3, -9, and IL-1ß in vitro; ii) determination of the effects of apelin by intra-articular injection with apelin and examination of MMP-3, -9, IL-1ß, collagen II, at both gene and protein levels in vivo. Furthermore, we analyzed the gene levels of ADAMTS-4, -5 and the proteoglycan content in articular cartilage in vivo. We demonstrated that: i) apelin $(0.5,1,10$, $100 \mathrm{nM})$ stimulated the proliferation of chondrocytes at any of these concentrations; ii) apelin $(0.5,1,10,100 \mathrm{nM})$ significantly increased the transcript levels of the catabolic factors MMP-1, -3, -9 and also the proinflammatory cytokine, IL-1ß in vitro; iii) in vivo, intra-articular injection with apelin increased the expression of MMP-3, -9, and IL-1ß as well as decreased the level of collagen II at both gene and protein levels. Additionally, after treatment of apelin, mRNA levels of ADAMTS-4 and -5 markedly increased and depletion of proteoglycan in articular cartilage was exhibited by histological assessment. Taken together, these findings indicate that apelin may play a risk role in the metabolism of chondrocytes and facilitated the progression of OA.

Many reports, concerned with the relationship between adipokines and OA have revealed that this novel family, including leptin, adiponectin, visfatin and resistin, play key roles in the pathophysiology of OA. Findings of Simopoulou et al show that leptin induces IL-1ß production and MMP-9, -13 protein expression, indicating that leptin acted as a proinflammatory role on cartilage metabolism (25). Lago et al demonstrated that adiponectin can induce expression of NOS2 and stimulate proinflammatory cytokine release, such as IL-6, MMP-3, -9 and monocyte chemoattractant protein-1 (MCP-1) on chondrocytes (8). Furthermore, that visfatin exerted a prodegradative effect by up-regulating the expression of MMP-3, -13 , ADAMTS -4 , and -5 and decreasing aggrecan production in chondrocytes, suggests that visfatin has a catabolic function in cartilage (10). Studies on different adipokines all revealed that they have played proinflammatory and catabolic/anabolic roles during the pathophysiology of OA. It is possible that apelin provides a metabolic link between obesity and OA.

In our study, apelin was observed to stimulate the proliferation of chondrocytes in vitro. Previous studied have proven that apelin can promote proliferation and suppress apoptosis of human osteoblasts $(15,22)$. Both chondrocytes and osteoblasts are derived from mesenchymal precursors and may share common signal transduction in cell proliferation. With this potential link, it is rather easy to understand its capability to stimulate the proliferation of chondrocytes. We also examined the effects of apelin on observed chondrocytes in monolayer cell culture. Interestingly, apelin at low concentrations $(0.5$ and $1 \mathrm{nM}$ ) remarkably induced the expression of MMP-1, -3, -9 and also the proinflammatory cytokine, IL-1ß. MMPs, whose levels were elevated in joint tissues of patients with rheumatic arthritis (RA) and OA, are considered as potential enzymes in the degeneration of cartilage $(26,27)$. MMP-1 belongs to the collagenase subclass and causes cleavage in type I, II, and III collagen. As a stromelysin, MMP-3 is well known to activate proMMP-1 by a cascade mechanism (28). It degrades several extracellular matrix molecules, including cartilage proteoglycan and type II collagen (29). MMP-9 degraded noncollagen matrix components of the joints (30). IL-1ß has been shown to stimulate the synthesis of MMPs, especially MMP-13, and induce the destruction of cartilage $(31,32)$. Our results indicate that apelin acts as a proinflammatory cytokine with a catabolic role on cartilage metabolism at lower concentrations in vitro. Meanwhile, our Western blotting results provided further 
evidence that apelin can stimulate protein expression of IL-1ß, MMP-3, -9, which were consistent with the date of real-time quantitative PCR.

A limitation of our in vitro study was that chondrocytes were cultured in monolayer. Therefore, local and systemic interferences could not be considered. To overcome these defects, we used an animal model in vivo for further research on the ability of proinflammatory and catabolic ability of apelin. The damage of the articular cartilage was mediated by the degradation of aggrecan and collagen II (33). Since apelin markedly increased the degradation enzymes such as MMP-1, -3 and -9 in vitro, we suspected that the component of aggrecan and collagen II would be down-regulated after intra-articular injection of apelin. Further in-depth studies were conducted on this catabolic ability in vivo.

We chose the concentration of apelin at $1 \mathrm{nM}$ for the intra-articular injection. Based on previous studies on intraarticular injection in rats, a $50-\mu 1$ solution was a reasonable amount in this animal model $(6,34)$. Since normal joint cavity of rat might contain a small amount of joint fluid, after intraarticular injection of $50 \mu \mathrm{l}$ of $1 \mathrm{nM}$ apelin, the final concentration of apelin in joint cavity would be $<1 \mathrm{nM}$, and might be close to $0.5 \mathrm{nM}$. From our results in vitro, apelin at $0.5 \mathrm{nM}$ was the most effective choice to induce catabolic and inflammatory roles.

Using intra-articular injection of apelin, we investigated the effect of apelin on the expression of collagen II, a major component of the extracellular matrix (ECM). Besides, we evaluated proteoglycan content in articular cartilage by Safranin O staining. We then evaluated degradation enzymes in vivo, including MMPs and ADAMTS families, which were viewed as key enzymes during the degeneration of articular (35). In our study, up-regulation of MMP-3, -9, and IL-1ß at both the mRNA and protein levels were found after apelin treatment in vivo, which was consistent with our previous study in vitro, indicating that the MMP family partly contributes to the catabolic ability of apelin. Furthermore, mRNA levels of ADAMTS-4 and -5 were significantly upregulated after treatment with apelin. ADAMTS-4 (also known as aggrecanase-1) and -5 (aggrecanase-2) were the most efficient aggrecanases which have been shown to cleave aggrecan at the aggrecanase cleavage site. Inhibition of ADAMTS-4 and -5 prevented aggrecan degradation in OA cartilage $(36,37)$. Double-knockout of ADAMTS-4 and -5 provided significant protection against proteoglycan degradation and decreased the severity of murine OA (38). This notably suggests that apelin is a disadvantageous factor involved in the process of OA not only by increasing MMPs in vivo, but also inducing aggrecanase production.

In summary, we firstly found that apelin promoted normal chondrocyte proliferation and induced expression of catabolic factors MMP-1, -3, -9, and IL-1ß in vitro and in vivo, as well as down-regulated the synthesis of collagen II. After apelin treatment, mRNA levels of ADAMTS-4 and -5 markedly increased and depletion of proteoglycan in articular cartilage was exhibited by histological assessment. Taken together, these findings clearly indicate that apelin acts as a catabolic factor involved in the progression of OA. This study also presented new findings on the relationship between obesity and OA and provided a new potential therapeutic target of OA. The specific mechanisms implicated in the regulation of apelin expression in the joint cavity and its signal transduction in the metabolism of chondrocytes should be further investigated.

\section{Acknowledgements}

This work was supported by the Health Bureau of Zhejiang Province (2006A055).

\section{References}

1. Felson DT, Chaisson CE, Hill CL, et al: The association of bone marrow lesions with pain in knee osteoarthritis. Ann Intern Med 134: 541-549, 2001.

2. Krasnokutsky S, Samuels J and Abramson SB: Osteoarthritis in 2007. Bull NYU Hosp Jt Dis 65: 222-228, 2007.

3. Felson DT, Anderson JJ, Naimark A, Walker AM and Meenan RF: Obesity and knee osteoarthritis. The Framingham Study. Ann Intern Med 109: 18-24, 1988.

4. Oliveria SA, Felson DT, Cirillo PA, Reed JI and Walker AM: Body weight, body mass index, and incident symptomatic osteoarthritis of the hand, hip, and knee. Epidemiology 10: 161-166, 1999.

5. Carman WJ, Sowers M, Hawthorne VM and Weissfeld LA: Obesity as a risk factor for osteoarthritis of the hand and wrist: a prospective study. Am J Epidemiol 139: 119-129, 1994.

6. Dumond H, Presle N, Terlain B, et al: Evidence for a key role of leptin in osteoarthritis. Arthritis Rheum 48: 3118-3129, 2003.

7. Chen TH, Chen L, Hsieh MS, Chang CP, Chou DT and Tsai SH: Evidence for a protective role for adiponectin in osteoarthritis. Biochim Biophys Acta 1762: 711-718, 2006.

8. Lago R, Gomez R, Otero M, et al: A new player in cartilage homeostasis: adiponectin induces nitric oxide synthase type II and pro-inflammatory cytokines in chondrocytes. Osteoarthritis Cartilage 16: 1101-1109, 2008.

9. Lee JH, Ort T, Ma K, et al: Resistin is elevated following traumatic joint injury and causes matrix degradation and release of inflammatory cytokines from articular cartilage in vitro. Osteoarthritis Cartilage 17: 613-620, 2009.

10. Gosset M, Berenbaum F, Salvat C, et al: Crucial role of visfatin/ pre-B cell colony-enhancing factor in matrix degradation and prostaglandin E2 synthesis in chondrocytes: possible influence on osteoarthritis. Arthritis Rheum 58: 1399-1409, 2008.

11. Lacinova Z, Michalsky D, Kasalicky M, et al: [The influence of obesity on the gene expression of adiponectin and its receptor in subcutaneous adipose tissue]. Vnitr Lek 53: 1190-1197, 2007.

12. De Falco M, De Luca L, Onori N, et al: Apelin expression in normal human tissues. In Vivo 16: 333-336, 2002.

13. O'Dowd BF, Heiber M, Chan A, et al: A human gene that shows identity with the gene encoding the angiotensin receptor is located on chromosome 11. Gene 136: 355-360, 1993.

14. Japp AG, Cruden NL, Amer DA, et al: Vascular effects of apelin in vivo in man. J Am Coll Cardiol 52: 908-913, 2008.

15. Xie H, Tang SY, Cui RR, et al: Apelin and its receptor are expressed in human osteoblasts. Regul Pept 134: 118-125, 2006.

16. Li F, Li L, Qin X, et al: Apelin-induced vascular smooth muscle cell proliferation: the regulation of cyclin D1. Front Biosci 13: 3786-3792, 2008 .

17. Reaux A, De Mota N, Skultetyova I, et al: Physiological role of a novel neuropeptide, apelin, and its receptor in the rat brain. J Neurochem 77: 1085-1096, 2001.

18. Cayabyab M, Hinuma S, Farzan M, et al: Apelin, the natural ligand of the orphan seven-transmembrane receptor APJ, inhibits human immunodeficiency virus type 1 entry. J Virol 74: 11972-11976, 2000.

19. Kleinz MJ and Davenport AP: Emerging roles of apelin in biology and medicine. Pharmacol Ther 107: 198-211, 2005.

20. Yonem A, Duran C, Unal M, Ipcioglu OM and Ozcan O: Plasma apelin and asymmetric dimethylarginine levels in type 2 diabetic patients with diabetic retinopathy. Diabetes Res Clin Pract 84: 219-223, 2009.

21. Han S, Wang GY, Qi X, Englander EW and Greeley GH: Involvement of a Stat 3 binding site in inflammation-induced enteric apelin expression. Am J Physiol-Gastrointest Liver Physiol 295: G1068-G1078, 2008.

22. Xie H, Yuan LQ, Luo XH, et al: Apelin suppresses apoptosis of human osteoblasts. Apoptosis 12: 247-254, 2007. 
23. Tang SY, Xie H, Yuan LQ, et al: Apelin stimulates proliferation and suppresses apoptosis of mouse osteoblastic cell line MC3T3E1 via JNK and PI3-K/Akt signaling pathways. Peptides 28 : 708-718, 2007.

24. Bao JP, Chen WP, Feng J, Hu PF, Shi ZL and Wu LD: Leptin plays a catabolic role on articular cartilage. Mol Biol Rep (In press).

25. Simopoulou T, Malizos KN, Iliopoulos D, et al: Differential expression of leptin and leptin's receptor isoform $(\mathrm{Ob}-\mathrm{Rb})$ mRNA between advanced and minimally affected osteoarthritic cartilage; effect on cartilage metabolism. Osteoarthritis Cartilage 15: 872-883, 2007.

26. Yoshihara Y, Nakamura H, Obata K, et al: Matrix metalloproteinases and tissue inhibitors of metalloproteinases in synovial fluids from patients with rheumatoid arthritis or osteoarthritis. Ann Rheum Dis 59: 455-461, 2000.

27. Dean DD, Martel-Pelletier J, Pelletier JP, Howell DS and Woessner JF Jr: Evidence for metalloproteinase and metalloproteinase inhibitor imbalance in human osteoarthritic cartilage. J Clin Invest 84: 678-685, 1989.

28. Murphy G, Cockett MI, Stephens PE, Smith BJ and Docherty AJ: Stromelysin is an activator of procollagenase. A study with natural and recombinant enzymes. Biochem J 248: 265-268, 1987.

29. Jo H, Park JS, Kim EM, et al: The in vitro effects of dehydroepiandrosterone on human osteoarthritic chondrocytes. Osteoarthritis Cartilage 11: 585-594, 2003.

30. Burrage PS, Mix KS and Brinckerhoff CE: Matrix metalloproteinases: role in arthritis. Front Biosci 11: 529-543, 2006.

31. Ijima $Y$, Kobayashi $M$ and Kubota E: Role of interleukin-1 in induction of matrix metalloproteinases synthesized by rat temporomandibular joint chondrocytes and disc cells. Eur J Oral Sci 109: 50-59, 2001.
32. Aida Y, Maeno M, Suzuki N, Shiratsuchi H, Motohashi M and Matsumura H: The effect of IL-1beta on the expression of matrix metalloproteinases and tissue inhibitors of matrix metalloproteinases in human chondrocytes. Life Sci 77: 3210$3221,2005$.

33. Kojima T, Mwale F, Yasuda T, Girard C, Poole AR and Laverty S: Early degradation of type IX and type II collagen with the onset of experimental inflammatory arthritis. Arthritis Rheum 44: 120-127, 2001.

34. Nakagawa S, Arai Y, Mazda O, et al: $\mathrm{N}$-acetylcysteine prevents nitric oxide-induced chondrocyte apoptosis and cartilage degeneration in an experimental model of osteoarthritis. J Orthop Res 28: 156-163, 2010.

35. Tortorella MD, Malfait AM, Deccico C and Arner E: The role of ADAM-TS4 (aggrecanase-1) and ADAM-TS5 (aggrecanase-2) in a model of cartilage degradation. Osteoarthritis Cartilage 9: 539-552, 2001.

36. Tortorella MD, Burn TC, Pratta MA, et al: Purification and cloning of aggrecanase-1: a member of the ADAMTS family of proteins. Science 284: 1664-1666, 1999.

37. Malfait AM, Liu RQ, Ijiri K, Komiya S and Tortorella MD: Inhibition of ADAM-TS4 and ADAM-TS5 prevents aggrecan degradation in osteoarthritic cartilage. J Biol Chem 277: 22201-22208, 2002.

38. Majumdar MK, Askew R, Schelling S, et al: Double-knockout of ADAMTS-4 and ADAMTS-5 in mice results in physiologically normal animals and prevents the progression of osteoarthritis. Arthritis Rheum 56: 3670-3674, 2007. 